\title{
In memoriam : André Constant
}

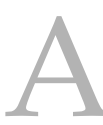

ndré Constant nous a quittés le 5 mai 2012; il fut un pilier de l'IRSID pendant les trente-cinq ans qu'il y a passé. Il est évidemment impossible de résumer en quelques mots sa carrière.

Si on se limite à quelques aspects, on se doit de rappeler ce qu'il a apporté de plus important à l'IRSID et à la sidérurgie, sur le plan des recherches et celui de la gestion des hommes.

Après ses études secondaires en Auvergne pendant la guerre et au lycée Hoche de Versailles, il entre à l'École des Mines de Paris en 1947 ; poussé par le professeur de métallurgie Paul Lacombe il entre en 1951 à l'IRSID, alors jeune institut tout juste sorti de terre et en plein recrutement. Sous la houlette de Georges Delbart la question principale était alors de concrétiser le programme de recherches en tenant compte des exigences nationales et des commettants.

À cette époque les demandes de recherches et développement concernaient des études générales, des mises au point de mesures et l'appui aux activités industrielles comme l'amélioration du procédé Thomas.

Parmi ce foisonnement André Constant choisit l'amélioration des connaissances de base des traitements thermiques des aciers alliés : il développa à cette fin avec son équipe des méthodes expérimentales éprouvées, pour tracer les courbes de transformation en refroidissement continu basées sur la dilatométrie en refroidissement continu et la métallographie.

En même temps que se constituait un riche atlas des diagrammes pour les aciers de construction, il eut l'idée d'appliquer ces techniques aux aciers dits courants ; cette idée s'est révélé un outil puissant d'étude et la combinaison avec les traitements thermomécaniques et l'emploi des éléments dispersoïdes, un ensemble métallurgique innovant, qui a permis à la sidérurgie française de prendre des parts de marché importantes dans les aciers à hautes caractéristiques.

Une autre vision claire des sujets de recherches fut de pressentir les besoins des utilisateurs que ce soit pour les aciers pour emplois à chaud, la mise en forme et la soudabilité ; cette dernière activité créée de toutes pièces avec Guy Murry et ensuite avec Gérard Bernard a porté de nombreuses avancées; il en fut de même avec l'introduction des études sur la rupture fragile et la mécanique de la rupture, études poursuivies par Germain Sanz.

Devenu adjoint au directeur des laboratoires, puis directeur de l'établissement, André Constant a vu son champ d'activités élargi : il a ainsi conforté plusieurs équipes en particulier la chimie physique de Paul Kozakevitch jusqu'à son départ à Mézières et contribué à la mutation de la chimie en l'orientant vers les problèmes de surfaces.

Il en fut de même plus tard pour les études sur les revêtements.

Les méthodes de travail d'André Constant furent très favorables à ses collaborateurs : recherche de la rigueur et beaucoup de liberté pour les chercheurs à condition de rester dans les limites.

Cet effort de formation a été multiplié par le rôle de pépinière qu'a joué l'IRSID durant cette période : pépinière pour les laboratoires des sociétés, pour les usines, pour les utilisateurs d'acier comme l'automobile, les tubistes etc., et même pour d'autres institutions comme le CEA, l'Euratom ... .

Il n'existe malheureusement pas de statistiques sur le nombre d'ingénieurs, de techniciens, de stagiaires qui sont passés par la formation qu'il avait encouragée.

Outre les nombreuses publications d'articles dans la revue de métallurgie et les revues étrangères il faut rappeler les trois atlas; courbes de transformation, fluage et soudabilité 
qui eurent une diffusion extrêmement large y compris à l'étranger et surtout l'ouvrage : «Bases métallurgiques des traitements thermiques » devenu ensuite: «Bases métallurgiques des traitements thermiques et thermomécaniques ».

La participation d'André Constant aux organismes de la sidérurgie (ATS, BNS, Chambre syndicale, CESSID) l'occupa beaucoup, et à l'extérieur il participa largement à l'Institut de Soudure et à l'IIS.

Dans la gestion de l'établissement il a souvent sauvegardé les embauches et les investissements dans des circonstances pas toujours faciles. J'ai personnellement beaucoup apprécié son soutien pour l'achat du laminoir à froid qui nous a offert de nouvelles perspectives.

Son rôle de directeur scientifique fut gêné par des ennuis de santé.

André Constant a reçu plusieurs distinctions en particulier les médailles Rist et Réaumur de la SF2M ainsi que le mérite national.

Le président et les membres de l'Association des Anciens de l'IRSID transmettent à sa famille l'expression de leur profonde émotion.

Marc Grumbach 